\title{
Impact of Motivation on Intentions in Online Learning: Canada vs China
}

\author{
Raafat George Saadé, Weiwei Tan, \& Fassil Nebebe \\ John Molson School of Business, Concordia University, \\ Montreal, Canada
}

\author{
rsinfo@sympatico.ca; ww tan@jmsb.concordia.ca; \\ fnebebe@jmsb.concordia.ca
}

\begin{abstract}
This study is one of the few empirical studies to examine students' acceptance of a web-based learning system (WLS). By incorporating intrinsic motivat or into the technology acceptance model, our model attempts to explain students' behavioral intention in using the system from a motivational perspective. Unlike most studies using subjects from developed countries, this study aims to extend knowledge by examining WLS adoption and usage behavior in a developing country (China) and then compares with findings of the same examination using Canadian subjects. The results show that both perceived usefulness and enjoyment have significant impact on students' intention to use WLS in two groups. However, in contrast to Chinese subjects, Canadian subjects do not think ease of use has impact on their intention to use WLS. Implications of this study are important for both researchers and practitioners, especially for institutions of higher education, planning to adopt WLS in the two countries.
\end{abstract}

Ke y words: Technology Accept ance Model, Web-based learning system, extrinsic motivation, intrinsic motivation, Developing country, China

\section{Introduction}

It is widely accepted today that the impact of Internet technologies is significant on every aspect of people's life. This impact is felt in the ever increasing pace of transformation of the higher education sector, while more and more institutions are using the internet and web technologies in the classroom as part of the learning environment (Piccoli, Ahmad, \& Ives, 2001). The advantages of internet-based learning mediums have been widely reported, recognized and accepted. Research suggests that technology-mediated learning environments allow for more study flexibility and broader accessibility (Lee, Cheung, \& Chen,, 2005), may improve students' performance (Alavi, 1994) and corresponding evaluation of their learning experiences (Hiltz, 1995), and lead to higher computer self-efficacy (Piccoli et al., 2001). In addition, it provides benefits to aca-

Material published as part of this publication, either on-line or in print, is copyrighted by the Informing Science Institute. Permission to make digital or paper copy of part or all of these works for personal or classroomuse is granted without fee provided that the copies are not made or distributed for profit or commercial advantage AND that copies 1) bear this notice in full and 2) give the full citation on the first page. It is permissible to abstract these works so long as credit is given. To copy in all other cases or to republish or to post on a serveror to redistribute to lists requires specific permission and payment of a fee. Contact Publisher@ InformingScience.org to request redistribution permission. demic institutions in terms of cost reductions and increased revenues (Saadé $\&$ Bahli, 2005) and has the potential of making learning more student-centered (Cardler, 1997).

In general, like any information systems, user acceptance and usage are important primary measures of system success (DeLone \& McLean, 1992). Without consideration of student involvement 
and participation, even the best developed system cannot be successful (Catchpole, 1993). Moreover, a successful web-base d learning system should be widely adopted with active participation from students. It is therefore import ant to identify, study and better understand the variables that influence learning in virtual environments and which lead to significant impact on student satisfaction, performance and adoption. Considering the literat ure in the past decade, the technology accept ance model (T AM), which is an adaptation of theory of reasoned action (TRA), seems to be dominant in the IS literature on explaining individuals' IT usage behavior (Lee et al., 2005; Straub, Keil, \& Brenner,, 1997; Taylor \& Todd, 1995).

The application of TAM has targeted mostly in the use of commercial soft ware, and recently the use of the internet/web. Very few studies however, applied the T AM to the online learning context in higher education. Moreover, the viability of T AM to explain individuals' accept ance behavior under the online learning context has been statistically confirmed in a recent study (Saadé, Nebebe, \& T an, 2007). Literat ure on T AM studies show that there is a dominant emphasis (of T AM) on notions of instrumentality, focusing mainly on functional or extrinsic motivational drivers (Agarwal \& Karahanna, 2000). In the context of student accept ance of web-based learning system (WLS), we believe intrinsic motivators representing a student's subjective feelings of joy, elation, pleasure, and positive holistic experience also play a critical role in explaining user acceptance and usage behavior of WLS. Researchers suggest that extrinsic and intrinsic motivat ors jointly determine the adoption of new technologies. Therefore, in this study, we postulate that incorporating both extrinsic (represented by perceived usefulness in TAM) and intrinsic motivation into TAM may enhance the explanation and prediction of student acceptance of WLS.

Furthermore, although many studies have examined user accept ance towards new technologies, most of them were conducted in developed countries using Western subjects, and thus on the whole may not be reflective of the adoption process in different cultural environments (Anandarajana, Igbaria, \& Anakwe, 2002; Straub et al., 1997). Relatively few T AM research studies have been done in Asian, Middle Eastern, and African countries and found none that were in the context on web-based learning. There are however some studies reported in the Informing Science Institute Conferences on experiences in online learning in Arab and Chinese countries, but these studies were descriptive in nat ure and reporting on process alone.

It is very important that TAM (and its variations) be applied in different countries because findings from prior studies support the import ance of culture as an influential factor impacting the existence and strength of relationships in theoretical models of technology acceptance behavior (Straub et al., 1997). In this study, in addition to investigating the intrinsic and extrinsic motivation constructs in TAM, we also apply the proposed research model to the culture in China. We therefore in this article compare the results bet ween the Chinese context and the Canadian Context. While the rate of using online learning system in higher education has been steadily increasing in China for the past decade, it is necessary to conduct research to get better understanding on Chinese students' behavior towards a WLS. Moreover, many Western universities provide distance learning programs supported by WLS in order to meet the increasing demands from Chinese education sector in recent years. How to successfully deliver these educat ional programs needs empirical studies to support local recommendations. In summary, the primary objective of this study is to empirically study and explain the impact of student motivation (intrinsic and extrinsic) on intentions while using a WLS.

The paper is organized as follows. The first section provides an overview of the theoretical background of the study. This is followed by a discussion of conceptual module and research hypotheses. The next section presents successively, research methodology, dat a analysis, and major findings of this study. Building upon these findings, practical and academic implications, as well as the limitations of the study, directions of future research are discussed. 


\section{Theoretical Background}

\section{Technology Acceptance Model and Extrinsic Motivation}

The theoretical grounding for this research is based on the technology acceptance model (F. D. Davis, 1989). In the T AM, F. D. Davis (1989) proposes that the influence of other variables on technology acceptance is mediated by two individual beliefs: perceived ease of use (PEU) and perceived usefulness (PU). In this study, perceived ease of use refers to the degree to which a student believes that using the WLS would be free from effort, whereas perceived usefulness is defined as the degree to which a student believes that using the WLS would enhance his or her performance in the course (F. D. Davis, 1989). Furthermore, PEU and PU will influence student's behavioral intention of using the WLS, leading to acceptance and adoption.

PU can be viewed as a measure of out come expectations. As such, PU of the WLS can lead to better grades. Similarly, PEU can be viewed as the extent to which the student expects the WLS to be easy to use, thereby making it less cognitively overloading thereby allowing students to feel more self efficient and direct most of their attention on achieving the expected tasks (F. D. Davis, 1989; Venkatesh, 1999). In this case PEU and PU can be considered as extrinsic motivational drivers.

Numerous studies have provided considerable support to the formulation of T AM under different technology contexts (Chau, 1996). Specifically, several studies about online learning affirm the validity of the influence of perceived ease of use and usefulness on student's intention. Saadé and Bahli (2005), for example, found that perceived usefulness of an internet-based learning system has a significant impact on behavioral intention, and that perceived ease of use is a predictor of perceived usefulness and intentions. Similar findings were replicated by Lee et al. (2005) in a study of investigating roles of motivation on intentions to use an internet-based learning medium; and by Yi \& Hwang (2003) in a study to extend the TAM in the context of a web-based class management system.

\section{Intrinsic Motivation}

Motivation theorists argue that motivation formulates the mechanism of human behavior and action. When a person is unmotivated, she/he would feel no impet us or inspiration to act. On the other hand, someone, who is energized or engaged on some activities toward an end, is considered motivated. It is not uncommon to view motivation as a unitary phenomenon in most theories of motivation, one that differentiates each other in levels. However, some researchers suggest that people have not only different amount, but also different orientations of motivation. They distinguish motivation to two broad classes, intrinsic and extrinsic motivation, according to the different reasons or goals to perform an action (Deci, 1975). Extrinsic motivation refers to the performance of an activity because it leads to instrumental rewards that are distinct from the activity it self (addressed in the previous section/paragraph). Intrinsic motivation refers to the performance of an activity for its inherent interests and enjoyment other than a separable outcome.

The T AM mainly emphasizes extrinsic perspective but lacks factors related to human and social change processes (Lee et al., 2005). With this dichotomy, only fairly recently have researchers begun to address the role of intrinsic motivation in studies in order to respond to the need of including more non-instrumental factors to T AM to provide broader view and a better explanation of IT adoption (e.g.Agarwal \& Karahanna, 2000; Heijden, 2003; Hsu \& Lu, 2004; Moon \& Kim, 2001; Saadé \& Bahli, 2005). Although the T AM emphasizes student behavior towards using WLS, it does not capture student's experiences while using the WLS (Lee et al., 2001). The capturing of intrinsic motivation constructs such as enjoyment while using the IT were found to be significant predictors of outcomes related to WLS use and accept ance (Ghani \& Deshpande, 
1994; Thompson et al., 1991). Although the T AM includes PU and PEU as extrinsic motivational drivers to explain acceptance, in the context of WLS, there is a need to consider a more complete view of motivation and expand the TAM to include intrinsic motivation (Saadé, 2007).

\section{Research Model and Hypotheses}

Figure 1 represents the theoretical model used in this study. This model integrates the motivational perspective into the TAM, and includes an intrinsic motivat or (enjoyment) as a salient determinant of student intention to use WLS.

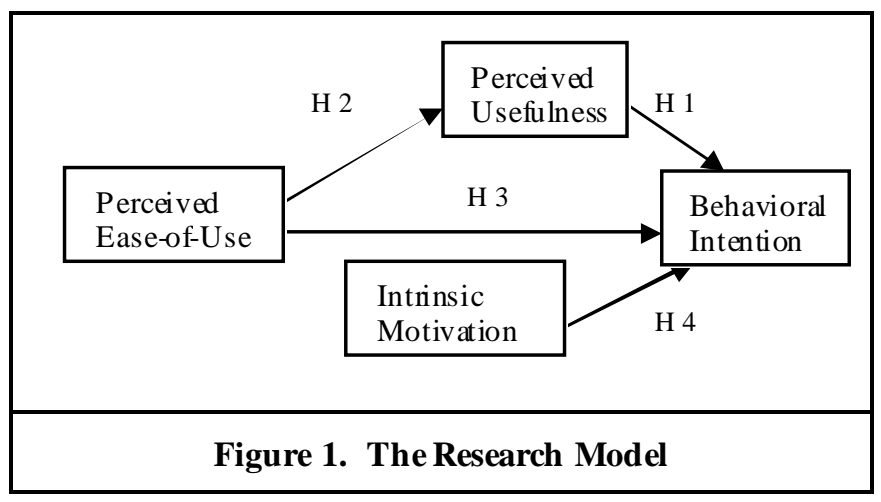

Perceived usefulness is defined as a student's expectation that using a particular system will result in improved course performance (F. D. Davis, 1989). From the motivational perspective, since an individual's behavior comes from his/her expectation on performance outcome, it represents an extrinsic fact or (F. D. Davis, Bagozzi, \& W arshaw, 1992). With the WLS students can access and download course materials freely and get online help from lecturers and other users in different locations via collaborative and community-building activities. Thus, it could be expected that students would believe in a use-performance relationship with the WLS, believing that if they use the WLS, their performance in the course would improve. We therefore hypothesize:

\section{H1: Perceived usefulness will have a positive influence on behavioral intention}

Based on Bandura's Social Cognitive Theory (1986), PEU inherits the notion of self-efficacy by capturing student's beliefs about their ability to successfully carry out a particular behavior. At the same time, due to the reduced cognitive burden (as a result of perceived ease of use) students may expect the instrumental award (PU) (F. D. Davis et al., 1992) of carrying out that behavior as elaborated in the Social Cognitive Theory (F. D. Davis, 1989; S. Davis \& Wiedenbeck, 2001). Consistent with the theoretical basis of TAM, we test the following hypothesis:

\section{H2: Perceived ease of use will have a positive influence on perceived usefulness}

Indeed, when students' assessment of their interaction with the system is relatively free of cognitive burden, they would logically have the intention to spend their time and effort to carry out the learning tasks. We thus hypothesize that:

\section{H3: Perceived ease of use will have a positive influence on behavioral intention}

Based on the intrinsic motivation theory, students would spend more time and efforts on a task, when this task raises a high level of enjoyment. In this study we use the enjoyment construct to conceptualize students' intrinsic motivation to use the WLS. Enjoyment refers to the extent to which the activity of using the computer is perceived to be enjoyable in its own right, apart from any performance consequences that may be anticipated (F. D. Davis et al., 1992). Venkatesh, Speier, and Morris (2002) suggest that intrinsically motivated computer users may tend to lower 
the perception of the difficulty associated with using a new technology, since enjoyment decreases the perception of effort on the use. Consistent with prior studies, we believe the relationship between enjoyment and behavioral intention should exhibit significant strength. Thus:

H4: Enjoyment has positive influence on behavioral intention of the WLS usage

\section{Research Method}

\section{Data Collection}

A survey methodology approach was followed in this study. A survey was administered at two universities, one in China and the other in Canada. In China, second year students of a business college taking a core course were asked to use a simple WLS to help them understand content material and rehearse for questions that may appear in the midterm and final exams. In Canada, undergraduate students of a business school in an introductory management information systems course were asked to use a similar online learning system to assist their study and assignments of an entry level IS course. Both of the WLSs were developed to be used via the web and students were able to use the learning tool anywhere, anytime. A total of 362 users in Canada and 120 students in China had completed the survey. These sets comprised the final sample used for data analysis.

\section{Instruments}

All the items used to measure the constructs were adapted from prior studies with modification to fit the specific context of the WLS. Both PU and PEU are measured by four items from F. D. Davis (1989), respectively, while two items are used to measure behavioral intention (Ajzen \& Fishbein, 1980). Enjoyment is measured using the scale adapted from F. D. Davis et al. (1992). All items used a five-point Likert-type scale with anchors from "Strongly disagree" to "Strongly agree" (see Appendix).

\section{Data Analysis}

The partial least-squares (PLS) approach to multiple indicat or structural equation analysis was used to assess the relationships between the constructs together with the predictive power of the research model. The PLS approach can be used for theory confirmation and to suggest where relationships might exist. For prediction, the PLS approach is more suitable than others, such as LISREL and EQS, because it assumes that all the measured variance in the study were to be explained. In behavioral research of the MIS field, PLS has been wholeheartedly accepted as an important statistical method (Goodhue, Lewis, \& Thompson, 2006). Accordingly, partial least squares via PLS-Graph (Chin, 2006) was used to analyze the datasets.

\section{Results}

We separately analyzed the two datasets in a two-step analytical procedure. We first examined the measurement model, and then the structural model. The measurement model using PLS was assessed in terms of convergent validity, internal consistency, and discriminant validity. The structural model and hypotheses were investigated by examining the path coefficients represented as standardized betas. The explained variance in the dependent constructs was assesse $d$ as an indication of the overall predictive strength of the model. 


\section{The Measurement Model}

Convergent validity represents the extent to which the indicators of a measurement are theoretically related and should correlate highly (Gefen \& Straub, 2005). A composite reliability of 0.7 or above and an average variance extracted (AVE) of greater than 0.7 are acceptable (Fornell \& Larcker, 1981). In addition, we introduced Cronbach's alpha to test reliability. All statistical measures were calculated for both datasets separately, since the data for each group is to be analyzed separately, and then compared. Table 1 summarizes the above parameters in our models for each group. All the measures fulfill the recommended levels, with the composite reliabilities higher than 0.9, AVE ranges from 0.70 to 0.89 . In both cases, alpha was greaterthan 0.7 for all constructs, thus demonstrating reliability.

\begin{tabular}{|l|c|c|c|c|c|c|}
\hline \multicolumn{7}{|c|}{ Table 1. Reliability and Convergent validity } \\
\hline \multirow{2}{*}{ Construct } & \multicolumn{2}{|c|}{ Composite Reliability } & \multicolumn{2}{c|}{ AVE } & \multicolumn{2}{c|}{ Cronbach Alpha } \\
\cline { 2 - 7 } & \multicolumn{2}{|c|}{ China (CN) } & Canada (CA) & \multicolumn{1}{c|}{ CN } & CA & \multicolumn{2}{c|}{ CN } & CA \\
\hline BI & 0.91 & 0.93 & 0.83 & 0.87 & 0.79 & 0.85 \\
\hline PU & 0.92 & 0.94 & 0.75 & 0.81 & 0.89 & 0.92 \\
\hline PEU & 0.90 & 0.94 & 0.70 & 0.89 & 0.86 & 0.88 \\
\hline EN & 0.92 & 0.94 & 0.79 & 0.79 & 0.86 & 0.91 \\
\hline
\end{tabular}

Discriminant validity is the extent to which the measure is not a reflection of some other variable. Discriminant validity is validated, when two thing happen: (1) the squared root of the average variance extracted for each construct is higher than the correlation between it and all other constructs (Fornell \& Larcker, 1981); and(2) if it ems have factor loading greater than 0.5 on their own construct, and much less than their loading on other constructs. When both cases occur then discriminant validity is supported (Gefen \& Straub, 2005). As summarized in Table 2 and Table 3 , all the measures are higher than the recommended levels.

\begin{tabular}{|l|c|c|c|c|}
\hline \multicolumn{5}{|c|}{ Table 2 Correlation of Latent Variables (China / Canada) group } \\
\hline & BI & PU & PEU & EN \\
\hline BI & $\mathbf{0 . 9 1 / 0 . 9 3}$ & & & \\
\hline PU & $0.80 / 0.45$ & $\mathbf{0 . 8 8 / 0 . 9 0}$ & & \\
\hline PEU & $0.73 / 0.27$ & $0.73 / 0.51$ & $\mathbf{0 . 8 4 / 0 . 9 4}$ & \\
\hline EN & $0.78 / 0.45$ & $0.83 / 0.61$ & $0.73 / 0.41$ & $\mathbf{0 . 8 9 / 0 . 8 9}$ \\
\hline
\end{tabular}

\begin{tabular}{|l|c|c|}
\hline \multicolumn{3}{|c|}{ Table 3. Factor Analysis (China / Canada) group } \\
& China & Canada \\
\hline BI1 & $\mathbf{0 . 9 1 8 6}$ & $\mathbf{0 . 9 4 4 4}$ \\
\hline BI2 & $\mathbf{0 . 9 0 0 8}$ & $\mathbf{0 . 9 2 4 6}$ \\
\hline PU1 & $\mathbf{0 . 8 2 6 7}$ & $\mathbf{0 . 8 9 7 4}$ \\
\hline PU2 & $\mathbf{0 . 8 7 3 5}$ & $\mathbf{0 . 9 0 3 5}$ \\
\hline PU3 & $\mathbf{0 . 8 7 7}$ & $\mathbf{0 . 9 2 2}$ \\
\hline PU4 & $\mathbf{0 . 8 9 0 1}$ & $\mathbf{0 . 8 8 7}$ \\
\hline
\end{tabular}




\begin{tabular}{|l|c|c|}
\hline PEU1 & $\mathbf{0 . 8 1 9 8}$ & $\mathbf{0 . 8 6 6 9}$ \\
\hline PEU2 & $\mathbf{0 . 8 5 0 1}$ & $\mathbf{0 . 8 7 2 8}$ \\
\hline PEU3 & $\mathbf{0 . 8 1 9 7}$ & $\mathbf{0 . 9 1 1}$ \\
\hline PEU4 & $\mathbf{0 . 8 5 9 9}$ & $\mathbf{0 . 9 0 4}$ \\
\hline EN1 & $\mathbf{0 . 8 6 2 5}$ & $\mathbf{0 . 9 4 2 7}$ \\
\hline EN2 & $\mathbf{0 . 8 9 5 8}$ & $\mathbf{- 0 . 7 7 7 7}$ \\
\hline EN3 & $\mathbf{0 . 9 0 5}$ & $\mathbf{0 . 9 4 8 4}$ \\
\hline
\end{tabular}

\section{The Structural Model}

The path coefficients from the PLS analysis are shown in Figure 2. As can be seen, the positive correlations between the constructs suggest that there are grounds for expecting significant effects bet ween them. PEU demonstrated direct, statistically significant, and positive effects on PU for both the China and Canada groups ( $\mathrm{path}=0.726$ and $0.511, \mathrm{p}<0.001$ ), respectively. As hypothesized, there is a strong positive relationship between PU and BI (path $=0.376$ and $0.271, p<0.01$ ) in both groups. The divergences between two datasets exist, when we calculate the influence of PEU on BI. It was found that PEU influences BI for the China group ( $p a t h=0.232, p<0.05$ ), but not for the Canada group ( $\mathrm{path}=0.012, \mathrm{p}=\mathrm{n} . \mathrm{s}$ ). However, the influence of enjoyment on user's BI for using WLS is weaker in China group ( $\mathrm{path}=0.303, \mathrm{p}<0.05$ ) than in Canada group ( $\mathrm{path}=0.282$, $\mathrm{p}<0.001$ ). For China group, $70.5 \%$ of the variance in BI was explained by models variables, $52.7 \%$ of the variance in PU is explained. For Canada group, both the percentages of the variance explained of BI and PU are much lower than those of China group, $25.2 \%$ and $26.1 \%$, respectively.

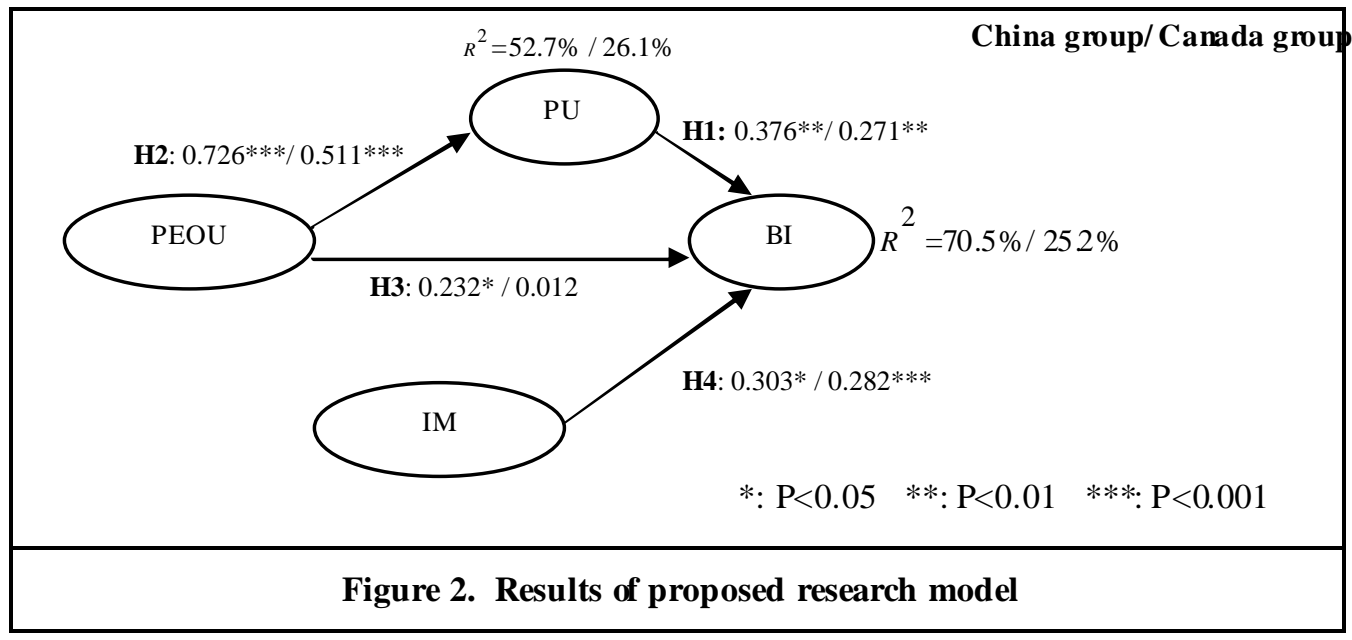

\section{Conclusions}

Motivated by a need to understand the underlying determinants of student adoption of WLS, our study included a motivational perspective into T AM, and postulated PU (extrinsic motivator) and Enjoyment (intrinsic motivat or) as the key drivers affecting students' acceptance behavior of WLS. For both subjects, the measurement model was confirmed with adequate convergent and discriminant validity with respect to the measurement of all the variables in the research model. In the structural model, all path coefficients were found statistically significant and strong, except 
the relationship between PEOU and BI in Canada group. On the other hand, it influences behavioral intention through PU.The results showed that PU and enjoyment have significant impact on student intention to use WLS.

The results of the empirical analysis provided many interesting insights and suggestions. First, the results indicated that (contrary to conventional prediction from T AM) PEU does not posit a significant direct effect on behavioral intention in Canada group but in China group. This observation is not sparse in recent studies conduced in developed countries (Saadé, 2003; Teo, Lim, \& Laia, 1999). For students in these countries, long time internet use makes them familiar to internet and web technologies, and thus learning to use the web-based study tools is generally considered easy. Ease of using the WLS has never really been a primary concern in their mind. Second, for both groups, the motivational factor seems to play an import ant role in explaining student acceptance of WLS. Individuals' intention to use the system increases, when they experience fun and enjoyment in the interaction with the WLS. Finally, compared with results of Canada group, PU and PEU in China group explained higher percent of variance in intention to use and have higher strength and significance on the relationships.

This study is one of the few attempts to investigate student acceptance of web-based learning environment. The use of student sample to measure an application designed for learning makes this study have a good fit between target technology and the study context. In addition, comparisons with the findings of different cultural samples help us better understand students' acceptance behavior. On the other side, the findings of this research must be considered in light of its limitations. First, the specific use of the WLS to practice certain study content would limit the user's value of the system. The student's value of the system largely depended on the importance individual students place on the task in preparing for their finals. Second, because of its specificity in terms of technology and culture, caution must be taken in generalizing and applying the results to other technologies and culture environments.

This article is motivated by a need to get a better understating of behaviors towards WLS. The findings demonstrate the essential value of Enjoyment and PU toward WLS in student acceptance behavior. From a practical perspective, this study contributes a theory-based empirical test to future WLS development. Chinese institutions can use the findings to underst and determinants that make students accept WLS for better performance in their studies. Western universities who wish to internationalize their programs could apply these to their distance learning program proposals.

\section{References}

Agarwal, R., \& Karahanna, E. (2000). Time flies when you're having fun: Cognitive absorption and beliefs about information technology usage. MIS Quarterly, 24(4), 665.

Ajzen, I., \& Fishbein, M. (1980). Understanding attitudes and predicting social behavior. NJ: PrenticeHall, Englewood Cliffs.

Alavi, M. (1994). Computer-mediated collaborative learning: An empirical evaluation. MIS Quarterly, $18(2), 159-174$.

Anandarajan a, M., Igbaria, M., \& Anakwe, U. P. (2002). IT acceptance in a less-developed country: A motivational factor perspective. International Journal of Information Management, 22, 47-65.

Bandura, A. (1986). Social foundations of thought and action: A social cognitive theory. Englewood Cliffs, NJ: Prentice-Hall.

Cardler, J. (1997). Summary of current research and evaluation of findings on technology in education. San Mateo, CA: Educational Support Systems.

Catchpole, M. J. (1993). Interactive media: The bridge between distance and classroom education. In T. Nunan (Ed.), Distance education futures (pp. 37-56). Adelaide: University of South Australia Press. 
Chau, P. Y. K. (1996). An empirical assessment of a modifi ed technology acceptance model. Journal of Management Information Systems, 13(2), 185.

Chin, W. W. (2006). PLS - Graph user's guide (Version 3.0) [Electronic Version].

Davis, F. D. (1989). Perceived usefulness, perceived ease of use, and user accept ance of in form ation technology. MIS Quarterly, 13(3), 319.

Davis, F. D., Bagozzi, R. P., \& Warshaw, P. R. (1992). Extrinsic and intrinsic motivation to use computers in the workplace. Journal of Applied Social Psychology, 22(14), 1111-1132.

Davis, S., \& Wiedenbeck, S. (2001). The mediating effects of intrinsic motivation, ease of use and usefulness perceptions on performance in first-time and subsequent computer users. Interacting with Computers, 13, 549-580.

Deci, E. L. (1975). Intrinsic motivation. New York: Plenum.

DeLone, W. H. \& McLean, E. R. (1992). Inform ation system success: The quest for the dependent variable. Information Systems Research, 3(1), 60-95.

Fornell, C., \& Larcker, D. F. (1981). Evaluating structural equations models with unobservable variables and measurement error. Journal of Marketing Research, 18(1), 39-50.

Gefen, D., \& Straub, D. (2005). A practical guide to factori al validity using PLS-Graph: Tutorial and annotated example. Communications of the Association for Information Systems, 16, 91-109.

Goodhue, D., Lewis, W., \& Thompson, R. (2006). PLS, Small sample size, and statistical power in MIS research. Paper presented at the the 39th Hawaii International Conference on System Sciences.

Heijden, H. V. D. (2003). Factors influencing the usage of websites: The case of a generic portal in The Netherlands. Information \& Management, 40(6), 541-549.

Hiltz, S. R. (1995). Teaching in a virtual classroom. International Journal of Educational Telecommunications, l(2), 185.

Hsu, C.-L., \& Lu, H.-P. (2004). Why do people play on-line games? An extended TAM with social influences and flow experience. Information \& Management, 41(7), 853-868.

Lee, M. K. O., Cheung, C. M. K., \& Chen, Z (2005). Acceptance of Intern et-bas ed learning medium: The role of extrinsic and intrinsic motivation. Information \& Management, 42(8), 1095-1104

Moon, J.-W., \& Kim, Y.-G. (2001). Extending the TAM for a World-Wide-Web context. Information \& Management, 38 (4), 217-230.

Piccoli, G., Ahmad, R., \& Ives, B. (2001). Web-based virtual learning environments: A research framework and a preliminary assessment of effectiveness in Basic IT Skills Training. MIS Quarterly, 25(4).

Saadé, R. (2003). Web-based educational information system for enhan ced learning, EISEL: Student assessment. Journal of Information Technology Education, 2, 267. Retrieved from http://iite.org/documents/Vol2/v2p267-277-26.pdf

Saadé, R., \& Bahli, B. (2005). The impact of cognitive absorption on perceived usefulness and perceived ease of use in on-line learning: an extension of the technology acceptance model. Information \& Management, 42(2), 261-386.

Saadé, R., Nebebe, F., \& Tan, W. (2007). Viability of the "Technology Acceptance Model" in multimedia learning environments: A comparative study. Interdisciplinary Journal of Knowledge and Learning Objects, 3, 175-184. Retrieved from http://ijello.org/Volume3/IJKLOv3p175-184Saade393.pdf

Straub, D. W., Keil, M., \& Brenner, W. H. (1997). Testing the technology acceptance model across cultures: A three country study. Information \& Management, 33, 1-11.

Taylor, S., \& Todd, P. A. (1995). Understanding information technology usage: A test of competing models. Information Systems Research 6(2), 144-176. 
Teo, T. S. H., Lim, V. K. G., \& Laia, R. Y. C. (1999). Intrinsic and extrinsic motivation in Internet usage. Omega, 27(1), 25-37.

Venkatesh, V., Speier, C., \& Morris, M. G. (2002). User acceptance en ablers in individual decision making about technology: Toward an integrated model Decision Sciences, 33(2), 297.

Yi, M. Y., \& Hwang, Y. (2003). Predicting the use of web-based in form ation systems: Selfefficacy, enjoyment, learning goal orientation, and the technology acceptance model. International Journal of Human-Computer Studies, 59, 431-449.

\section{Appendix: Scales and Items}

\begin{tabular}{|c|c|c|}
\hline Construct & Item & Measure \\
\hline \multirow[t]{4}{*}{ Perceived Usefulness (PU) } & PU1 & $\begin{array}{l}\text { Using the web-based learning system would improve my } \\
\text { perform ance in the course }\end{array}$ \\
\hline & PU2 & $\begin{array}{l}\text { Using the web-based learning system in the course would } \\
\text { increase my productivity }\end{array}$ \\
\hline & PU3 & $\begin{array}{l}\text { Using the web-based learning system would enhance my } \\
\text { effectiveness in the course }\end{array}$ \\
\hline & PU4 & $\begin{array}{l}\text { I would find the web-based learning system useful in } \\
\text { study }\end{array}$ \\
\hline \multirow[t]{4}{*}{ Perceived Eas e of Use (PEU) } & PEU1 & $\begin{array}{l}\text { Learning to operate the web-based learning system would } \\
\text { be easy for me }\end{array}$ \\
\hline & PEU2 & $\begin{array}{l}\text { I would find it easy to get the web-based learning system } \\
\text { to do what I want it to do }\end{array}$ \\
\hline & PEU3 & $\begin{array}{l}\text { It would be easy for me to become skillful at using the } \\
\text { web-bas ed learning system }\end{array}$ \\
\hline & PEU4 & I would find the web-based learning system easy to use \\
\hline \multirow[t]{2}{*}{ Behavioral Intention (BI) } & BI1 & $\begin{array}{l}\text { I intend to take more courses using the web-based learning } \\
\text { system in the future. }\end{array}$ \\
\hline & $\mathrm{BI} 2$ & I intend to show others this web-based learning system. \\
\hline \multirow[t]{3}{*}{ Enjoyment } & EN1 & I find the web-based learning system was enjoyable \\
\hline & EN2 & $\begin{array}{l}\text { The actual process of using the web-based learning system } \\
\text { was pleasant }\end{array}$ \\
\hline & EN3 & I had fun using the web-based learning system \\
\hline
\end{tabular}

\section{Biographies}

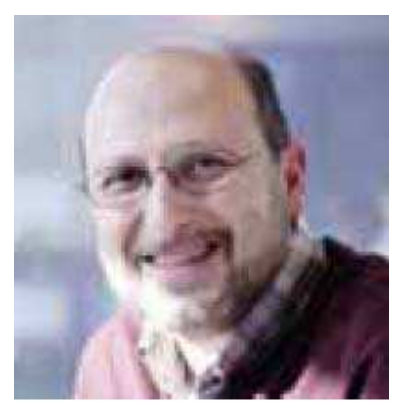

Dr. Raafat Saadé is a lecturer at the DSMIS department, John Molson School of Business, Concordia University, Canada. Dr. Saadé obtained his Ph.D. in 1995 from Concordia University. He subsequently received the Canadian National Research Council postdoctoral fellowship, which he completed at McGill University (Montreal). He has been recognized twice as a North Atlantic Treaty Organization ASI award winner. Dr. Saadé has 18 years of industrial experience (engineering, elearning and ehealth), and presently is involved in international consulting projects. He is very active in research with over 25 peer refereed journal articles. Dr. Saadé has published in top tier journals including Information \& Management, JISE, Expert Systems with Applications, and Decision Sciences. 


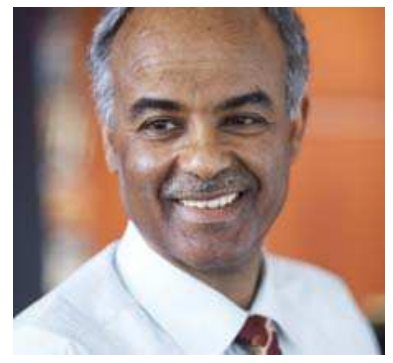

Dr. Fassil Ne be be is a Full Professor at the Department of Decision Sciences \& M.I.S. of the John Molson School of Business, Concordia University, Montreal, Canada. His research interests are in Bayes and empirical Bayes modeling and analysis of dat a, resampling methods, small area estimation, statistical computing and data mining. He has served as Managing Edit or of Liaison - The Newsletter of the Statistical Society of Canada and the President of the Statistical Society of Montreal. He is the founding President of the SSENA, and since 1995 he has been an International Advisory Board member of SINET: Ethiopian Journal of Science, and JESA: Journal of the Ethiopian Statistical Association.

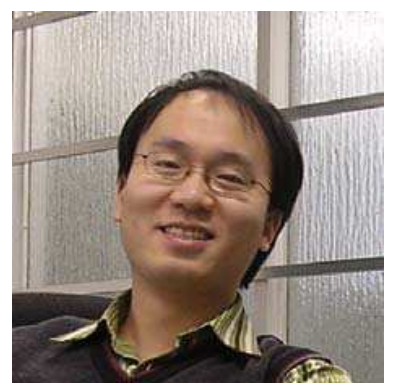

Mr. Weiwei Tan is an M.SC graduate from Concordia University, John Molson School of Business. He is presently a research assistant in the department of Decision Sciences and MIS. His research interests are in the adoption, implement ation and use of information technologies, and the business value and intelligence of Internet-based activities on individuals and organizations. 